\title{
Enumeration of Specific Classes of Polycubes
}

\author{
Jean-Marc Champarnaud \\ LITIS \\ Université de Rouen \\ France \\ jean-marc . champarnaud@univ-rouen.fr \\ Jean-Philippe Dubernard \\ LITIS \\ Université de Rouen \\ France \\ jean-philippe.dubernard@univ-rouen.fr
}

\author{
Quentin Cohen-Solal \\ LITIS \\ INSA Rouen \\ France \\ quentin.cohensolal@insa-rouen.fr \\ Hadrien Jeanne \\ LITIS \\ Université de Rouen \\ France \\ hadrien.jeanne@univ-rouen.fr
}

Submitted: Jul 7, 2013; Accepted: Nov 20, 2013; Published: Nov 29, 2013

Mathematics Subject Classifications: 05A15, 05A16

\begin{abstract}
The aim of this paper is to gather several new results concerning the enumeration of specific classes of polycubes. We first consider two classes of 3-dimensional vertically-convex directed polycubes: the plateau polycubes and the parallelogram polycubes. An expression of the generating function is provided for the former class, as well as an asymptotic result for the number of polycubes of each class with respect to volume and width. We also consider three classes of $d$-dimensional polycubes $(d \geqslant 3)$ and we state asymptotic results for the number of polycubes of each class with respect to volume and width.
\end{abstract}

\section{Introduction}

In the cartesian plane $\mathbb{N}^{2}$, a polyomino is a finite connected union of elementary cells (unit squares) without cut points and defined up to a translation. Even though polyominoes have been studied for a long time in combinatorics, no exact formula is known for general polyominoes but many results have been found concerning some classes of polyominoes, see for instance [6] or [13].

Polyominoes also have a 3-dimensional equivalent: the polycubes [17]. If we consider, now, that an elementary cell is a unit cube, then a polycube is a face-connected finite set 
of elementary cells defined up to a translation in $\mathbb{N}^{3}$. As with polyominoes, polycubes appear in statistical physics, more precisely in the phenomenon of percolation (see [5] for example). A lot of studies have led to count polycubes according to the number $n$ of cells composing them. The first values were found in 1972 up to $n=6$ [17] and the last one (to our knowledge) in 2006, up to $n=18$ [2]. If we extend the notion of polycube to $d$-dimensions, where $d \geqslant 3$, these objects are also used in an efficient model of real-time validation [16], as well as in the representation of finite geometrical languages [8, 14, 12].

However a small number of families of polycubes have been investigated. The most well-known, for which there exists a rich literature, is the one of plane partitions (see $[3,11,4,19]$ for instance). Recently, a new method has allowed us to enumerate several families of polycubes (in dimension 3 and greater than 3) as far as they can be split into strata $[10,14,12]$. It is worthwhile noticing that it is the investigation of the families of plateau and parallelogram polycubes (as reported in [9]) that motivated the design of this method.

In the next section, following the model of polyominoes, we define several classes of polycubes and we recall a method, due to M. Bousquet-Mélou [6], for the enumeration of classes of column-convex polyominoes, that we will use to enumerate plateaus. Then, in Section 3, we present the results obtained for the enumeration of a particular class of vertically-convex polycubes (the plateau ones). Partial results for one subclass (parallelogram polycubes) of the class of plateau polycubes are reported in Section 4 . The last section contains asymptotic results concerning $d$-dimensional polycubes $(d \geqslant 3)$ that can be deduced from results of Sections 3 and 4 and that complete those found in [10].

\section{Preliminaries}

\subsection{Polycubes}

A polyomino is said to be row-convex (resp. column-convex) if its intersection with any horizontal (resp. vertical) strip is convex. It is said to be directed if each of its cells can be reached from a distinguished cell, called the root, by a path only made of East and North steps.

Let $(0, \vec{i}, \vec{j}, \vec{k})$ be an orthonormal coordinate system where $\vec{i}$ and $\vec{k}$ are horizontal vectors and $\vec{j}$ the only vertical vector.

As for polyominoes, several parameters can be defined for a polycube. The volume is the number of elementary cells, the width (resp. height, depth) is the difference between the greatest and the smallest indices of the polycube according to $\vec{i}$ (resp. $\vec{j}, \vec{k}$ ). A polycube is said to be horizontally-convex if its intersection with any horizontal plane $(\vec{i}, \vec{k})$ is a row-convex polyomino; it is said to be vertically-convex if its intersection with any vertical plane $(\vec{i}, \vec{j})$ is a column-convex polyomino. An example of horizontally-convex polycube is given in Figure 1. Similarly, a polycube is said to be antihorizontally-convex if its intersection with any horizontal plane $(\vec{i}, \vec{k})$ is a column-convex polyomino; it is said to be antivertically-convex if its intersection with any vertical plane $(\vec{j}, \vec{k})$ is a column-convex polyomino. 

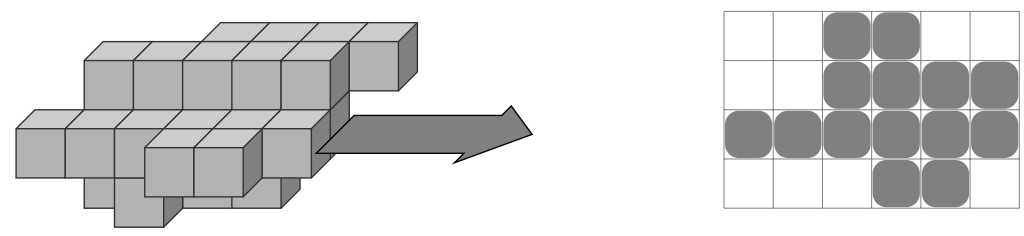

Figure 1: A horizontally-convex polycube.

A polycube is said to be convex if it is both horizontally, antihorizontally, vertically and antivertically convex. The 2-dimensional notion of step is extended as follows. An East (resp. North, Ahead) step is a move of one unit in the $\vec{i}$-direction (resp. $\vec{j}$-direction, $\vec{k}$-direction). So, a polycube is said to be directed if each of its cells can be reached from a distinguished cell, called the root, by a path only made of East, North and Ahead steps.

The 2-dimensional notion of column is extended as follows. A stratum is a polycube of width 1 . Hence a stratum can be directed or horizontally (resp. vertically, antihorizontally, antivertically) convex. Two examples of strata are given in Figure 2. The notion of stratum allows us to define two new families of polycubes.

A polycube is said to be $s$-directed if the two following conditions are satisfied:

(1) its strata are directed,

(2) its strata are glued together so that the polycube is directed.

A polycube is said to be vertically convex s-directed if the two following conditions are satisfied:

(1) its strata are vertically convex directed,

(2) its strata are glued together so that the polycube is directed.

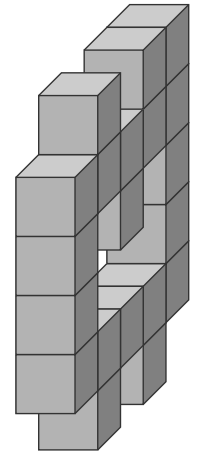

(1)

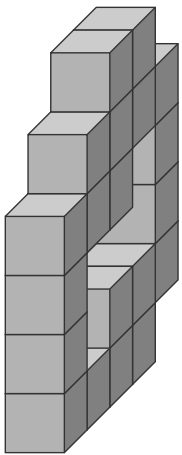

$(2)$

Figure 2: An ordinary stratum (1) and a directed stratum (2).

A particular vertically convex s-directed polycube is the plateau polycube that can be obtained by gluing together vertical plateaus.

Intuitively, we define the front (resp. the back) of a polycube as the closest (resp. the furthest) side of the plane $(O, \vec{i}, \vec{j})$. The bottoms and the tops of a polycube are defined 

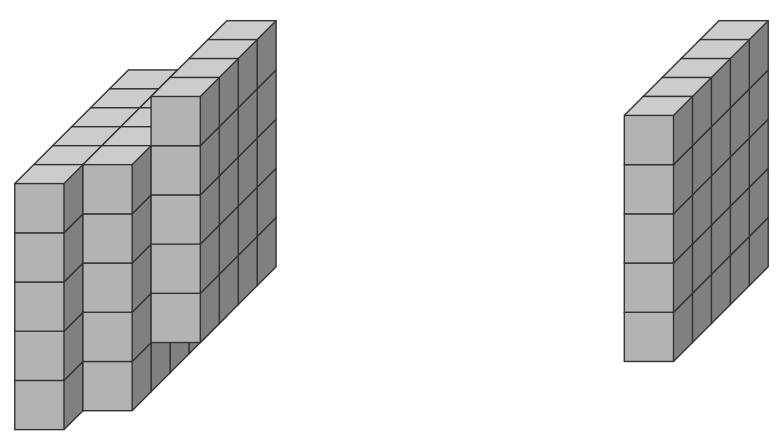

Figure 3: A plateau polycube of width 3 and volume 75 and a plateau of volume 25 .

similarly as for a polyomino. A parallelogram polycube is a plateau polycube such that each vertical plateau has no vertical plateau to its right and behind it that gets a cell under its bottom neither to its left and in front of it that gets a cell above its top. Note that this definition implies that the projection of a parallelogram polycube w.r.t. $(O, \vec{i}, \vec{j})$ (resp. w.r.t. $(O, \vec{j}, \vec{k}))$ gives a parallelogram polyomino.

\subsection{Enumeration}

Let $\mathcal{R}=\mathbb{R}[[s, x, q]]$ be the algebra of formal power series in the variables $s, x, q$ and with real coefficients and let $\mathcal{A}$ be a sub-algebra of $\mathcal{R}$ such that the series are convergent for $s=1$. If $X(s, x, q)$ is such a series, we will often denote it $X(s)$. Its derivative, with respect to $s$ will be denoted $X^{\prime}(s)$.

In [6], Bousquet-Mélou uses the decomposition of column-convex polyominoes to express functional equations that have the same form for any class of convex polyominoes. She also proves the following lemma to solve this type of equation:

Lemma 1. [6] Let $X(s)$ be a formal power series lying in $\mathcal{A}$. Suppose that:

$$
X(s)=x e(s)+x f(s) X(1)+x g(s) X(s q),
$$

where $e(s), f(s)$ and $g(s)$ are some given power series in $\mathcal{A}$. Then

$$
X(1)=\frac{E(1)}{1-F(1)}
$$

where

$$
E(s)=\sum_{n \geqslant 0} x^{n+1} g(s) g(s q) \ldots g\left(s q^{n-1}\right) e\left(s q^{n}\right)
$$

and

$$
F(s)=\sum_{n \geqslant 0} x^{n+1} g(s) g(s q) \ldots g\left(s q^{n-1}\right) f\left(s q^{n}\right)
$$



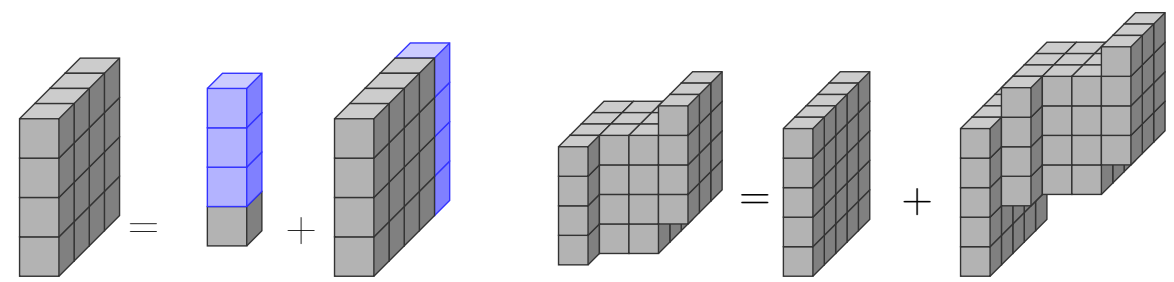

Figure 4: Decomposition of plateaus and plateau polycubes.

\section{Enumeration of plateau polycubes}

A plateau polycube can be split into plateaus that are glued together. So, we need, at first, to find an expression of the generating function of plateaus with respect to height, volume and area of the rightmost face. Next, we use this result to enumerate plateau polycubes.

\subsection{Case of plateaus}

Let $P(s, p, q)$ be the generating function of vertical plateaus with respect to height (coded by $s$ ), volume (coded by $p$ ) and surface of the rightmost face (coded by $q$ ). Notice that the volume of a plateau is equal to the area of its rightmost face. However, as we need the area later, we simultaneously consider the two parameters. Let us remark that our method is inspired by the one described in [6] to enumerate various classes of column-convex polygons.

To enumerate vertical plateaus, we have to consider two different cases (see Figure 4):

- the columns (vertical plateaus of depth 1): their generating function is $\frac{s p q}{1-s p q}$.

- the plateaus of depth $>1$ : such plateaus are obtained by gluing a new column behind a plateau of depth $\geqslant 1$; they are enumerated by $P(s p q, p, q)$.

Hence the following proposition:

Proposition 2. The generating function $P(s, p, q)$ of plateaus satisfies the functional equation:

$$
P(s, p, q)=\frac{s p q}{1-s p q}+P(s p q, p, q)
$$

Using the Lemma 1, we obtain the following proposition:

Proposition 3. The generating function $P(s, p, q)$ of plateaus satisfies:

$$
P(1, p, q)=\sum_{n \geqslant 1} \frac{(p q)^{n}}{1-(p q)^{n}} .
$$


Let $P(1, p, 1)=\sum_{n \geqslant 1} f_{n} p^{n}$; we can remark that $f_{n}=\tau(n)$ is the number of divisors of $n$ [20]. Finding a bijection between these two families is trivial. For instance, we just have to consider that a divisor $d$ of $n$ is in bijection with the plateau of volume $n$ and height $d$. Notice also that there is no explicit formula for $\tau(n)$.

\subsection{Case of plateau polycubes}

The idea is to decompose these polycubes in vertical plateaus. Let $G(t, p)$ be their generating function with respect to width and volume. We have to consider two cases to enumerate them:

- the plateau polycubes are plateaus: using proposition 2, we immediately find that their generating function is $t P(1, p, 1)$;

- they can be split into a plateau on which we glue a plateau polycube: notice that the South-West corner of the polycube must be glued on a cell of the right face of the plateau; their generating function is $t\left(\frac{\partial P}{\partial q}\right)(1, p, 1) G(t, p)$.

Finally, we obtain the following equation:

$$
G(t, p)=t P(1, p, 1)+t\left(\frac{\partial P}{\partial q}\right)(1, p, 1) G(t, p)
$$

and the following proposition:

\section{Proposition 4.}

$$
G(t, p)=\frac{t \sum_{k \geqslant 1} \frac{p^{k}}{1-p^{k}}}{1-\sum_{k \geqslant 1} \frac{k t p^{k}}{\left(1-p^{k}\right)^{2}}} .
$$

From Proposition 4, it is easy to obtain the first values of $G(t, p)$. They are given in Table 1. From these values, it has been possible to find some properties about $G(t, p)$. Let us set $G(t, p)=\sum_{n, m} g_{n, m} t^{n} p^{m}$ where $g_{n, m}$ is the number of plateau polycubes of width $n$ and volume $m$.

\section{Proposition 5.}

1. $g_{1, n}=\tau(n)$;

2. $g_{n, n}=1$;

3. $g_{n-1, n}=4 n-6$;

4. $g_{n-2, n}=8 n^{2}-42 n+56$;

5. $g_{n-3, n}=\frac{32}{3} n^{3}-120 n^{2}+\frac{1384}{3} n-605$. 
Table 1: The first values of $g_{n, m}$.

\begin{tabular}{l|cccccccccccc}
$\mathrm{p} \backslash \mathrm{t}$ & 1 & 2 & 3 & 4 & 5 & 6 & 7 & 8 & 9 & 10 & 11 & 12 \\
\hline 1 & 1 & & & & & & & & & & & \\
2 & 2 & 1 & & & & & & & & & \\
3 & 2 & 6 & 1 & & & & & & & & \\
4 & 3 & 16 & 10 & 1 & & & & & & & \\
5 & 2 & 35 & 46 & 14 & 1 & & & & & & \\
6 & 4 & 60 & 147 & 92 & 18 & 1 & & & & & \\
7 & 2 & 98 & 378 & 403 & 154 & 22 & 1 & & & & \\
8 & 4 & 148 & 824 & 1372 & 867 & 232 & 26 & 1 & & & \\
9 & 3 & 198 & 1638 & 3894 & 3714 & 1603 & 326 & 30 & 1 & & \\
10 & 4 & 290 & 2948 & 9680 & 13068 & 8332 & 2675 & 436 & 34 & 1 & & \\
11 & 2 & 352 & 5029 & 21726 & 39594 & 35178 & 16410 & 4147 & 562 & 38 & 1 & \\
12 & 6 & 480 & 8100 & 44836 & 106740 & 126540 & 81152 & 29388 & 6083 & 704 & 42 & 1
\end{tabular}

Proof. The proofs of these formulas are based on a unique principle. We start from the plateau of height 1 , depth 1 and width $n-i, i \leqslant 3$ (and volume $n-i$ ). Next, we build all the polycubes of width $n-i$ and volume $n$ from this plateau by adding $i$ cells, cutting and gluing it. Thus, we only detail the proof of the value of $g_{n-2, n}$. Let us consider the polycube of volume $n-2$ and width $n-2$ and let us enumerate all the ways to inject two cells to obtain a plateau polycube. There are two main cases :

- the two cells are inserted on the same plateau:

- if we insert the cells on the last plateau, as in Figure 5, we have two possibilites, vertically or horizontally;
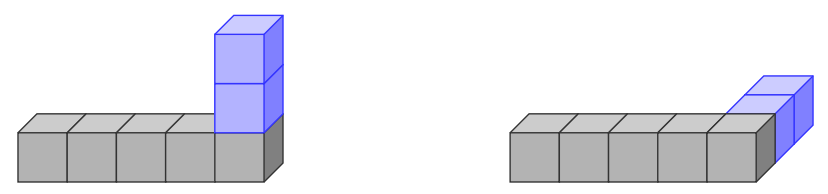

Figure 5: Insertion on the last plateau.

- otherwise, for a given column, there are six ways to insert two cells : three vertical insertions (see Figure 6) and three horizontal ones. As we can choose any of the $n-3$ columns, we obtain $6(n-3)$ possibilities.

- the cells are inserted on two different plateaus, as in Figure 7:

- one of the two cells is on the last plateau and there are two different ways to glue it. The second cell is then added to one of the $n-3$ other plateaus. Since we have four different ways to inject the cell, we obtain $8(n-3)$ possibilities; 

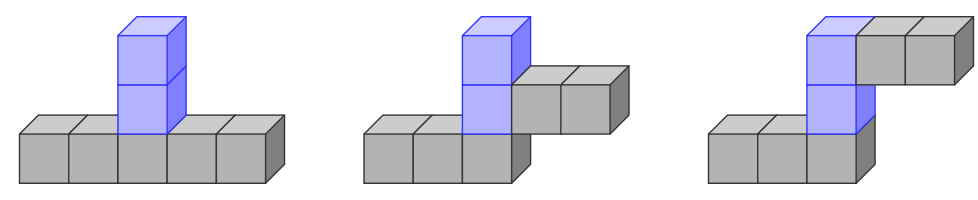

Figure 6: Insertion on a plateau different of the last one.

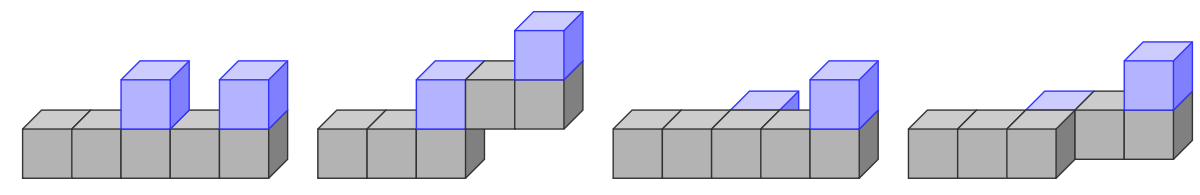

Figure 7: Insertion on two different plateaus.

- in the case where no cell is added to the last plateau, we have to choose 2 plateaus among $n-3$, what gives $\left(\begin{array}{c}n-3 \\ 2\end{array}\right)$ possibilities. Moreover, we have already established that there are 4 different ways to add a cell to a plateau. Since this operation must be applied on two different plateaus, we obtain $4^{2}\left(\begin{array}{c}n-3 \\ 2\end{array}\right)$ possibilities.

When we add all the possibilities we finally find: $g_{n-2, n}=8 n^{2}-42 n+56$.

Notice that this proof is similar as the ones established in [10] in the case of the enumeration concerning families of 3-dimensional polycubes. Moreover, it is not possible to find a formula in the general case because it depends on the $\tau(k)$ coefficients that do not have explicit formula. However, it is possible to state the following proposition:

Proposition 6. $g_{n-i, n}$ is a polynomial in $\mathbb{Q}[n]$ whose highest-degree-term is equal to $\frac{4^{i}}{i !} n^{i}$.

Proof. The proof is based on the same principle as in Proposition 5. Let us consider the plateau of height 1 , depth 1 and width $n-i$ and let us add to it $i$ cells on $i$ different columns (except for the last one). We have $\left(\begin{array}{c}n-i-1 \\ i\end{array}\right)$ different choices of columns. Then, for each selected column, we have $2 * 2$ possible constructions to obtain a polycube. As we perform this construction on $i$ columns, we finally obtain $4^{i}\left(\begin{array}{c}n-i-1 \\ i\end{array}\right)$ possibilities.

In all the other cases, we only have $\left(\begin{array}{c}n-i-1 \\ i-j\end{array}\right), j>1$, possibilities to choose columns, leading to a term of degree $i-j$. Since $j>1$, the highest-degree-term is in $O\left(n^{i-j}\right)$, which is unimportant in front of $O\left(n^{i}\right)$. So, the highest degree term in $n$ is $\frac{4^{i}}{i !} n^{i}$.

Computing a polynomial interpolation of $G(t, p)$ leads to the following results: 


\section{Corollary 7.}

$$
\begin{aligned}
g_{n-3, n} & =\frac{32}{3} n^{3}-120 n^{2}+\frac{1384}{3} n-605 \\
g_{n-4, n} & =\frac{32}{3} n^{4}-208 n^{3}+\frac{4678}{3} n^{2}-5324 n+6972 \\
g_{n-5, n} & =\frac{128}{15} n^{5}-256 n^{4}+3144 n^{3}-19756 n^{2}+\frac{951742}{15} n-83160 \\
g_{n-6, n} & =\frac{256}{45} n^{6}-\frac{1216}{5} n^{5}+\frac{39824}{9} n^{4}-\frac{131572}{3} n^{3}+\frac{11231344}{45} n^{2}-\frac{11583502}{15} n \\
& +1013028 \\
g_{n-7, n} & =\frac{1024}{315} n^{7}-\frac{2816}{15} n^{6}+\frac{213184}{45} n^{5}-\frac{203152}{3} n^{4}+\frac{26635408}{45} n^{3}-\frac{47411774}{15} n^{2} \\
& +\frac{333749548}{35} n-12517020 \\
g_{n-8, n} & =\frac{512}{315} n^{8}-\frac{2560}{21} n^{7}+\frac{183232}{45} n^{6}-\frac{237344}{3} n^{5}+\frac{43996894}{45} n^{4}-7864440 n^{3} \\
& +\frac{12654017396}{315} n^{2}-\frac{832930465}{7} n+156257400
\end{aligned}
$$

\section{Some propositions about parallelogram polycubes}

Let us establish a functional equation satisfied by the generating function of parallelogram polycubes with respect to several parameters. We first introduce the following variables:

- $p$ that encodes the volume of the polycube;

- $t$, its width;

- $q$, the area of the rightmost face of the polycube;

- $h$, the heigth of the last plateau;

- $r$, the depth of the last plateau.

Let us note $H(t, h, r, p, q)=\sum_{n, m} h_{n, m} t^{n} p^{m}$, the generating function of parallelogram polycubes with respect to the above-mentioned parameters. Let us notice that these polycubes appear in the modelling of real-time applications composed of two periodic tasks [15].

Let us consider a parallelogram polycube. Then, several cases can appear:

- The first case (decomposition 2 in Figure 8) is the one where it is reduced to a plateau. Thus, it is enumerated by $P l(t, h, r, p, q)$. 

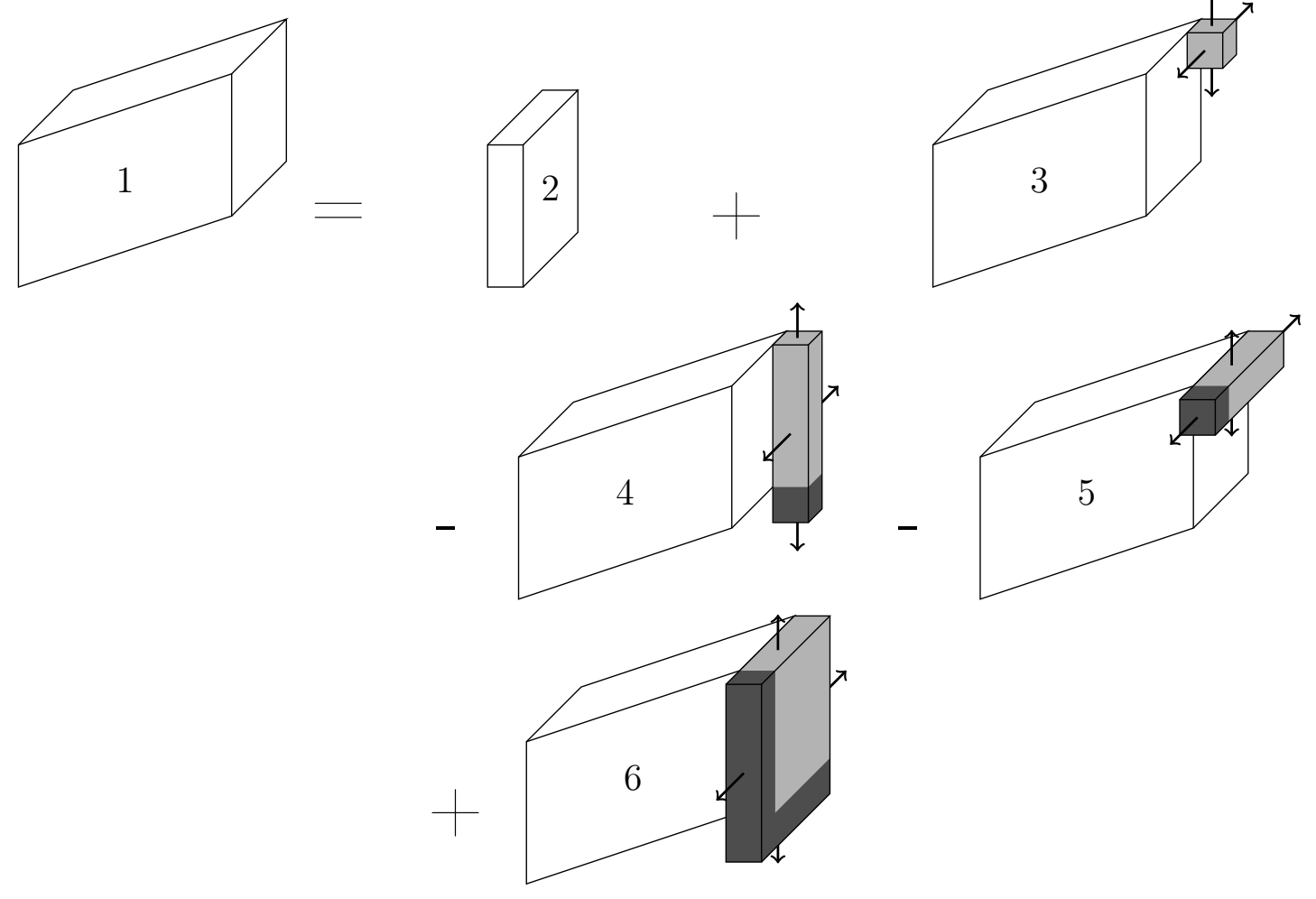

Figure 8: Decomposition of the construction of a parallelogram polycube.

- Otherwise, the parallelogram polycube can be built from a smaller polycube, adding to it a new plateau. We start by gluing a cell at the top and at the bottom of the rightmost plateau (decomposition 3 in Figure 8). We add as many cells as we want on the top and under the bottom. Then, we duplicate this column upward and downward to build the last plateau.

Let us translate this construction into a generating function. All the possibilities are enumerated by $H(t, h, r, p, q)$.

We begin by gluing a cell at the top and at the bottom of the rightmost plateau. Adding a new cell leads to the creation of a new plateau and the new cell is coded by pqrh. The constructions that we obtain are enumerated by $H(t, 1,1, p, 1)$ pqrth .

Then, we make the cell grow up and down. Adding a cell amounts to an increase of 1 of the area, the volume and the height of the rightmost plateau, which leads to a factor $p q h$. Adding as many cells as possible upward is enumerated by:

$$
1+p q h+(p q h)^{2}+\ldots+(p q h)^{n}=\frac{1}{1-p q h} .
$$

A similar reasoning for downward growth leads to a factor $\frac{1}{(1-p q h)^{2}}$. Therefore we get the following generating function: 


$$
H(t, 1,1, p, 1) \frac{p q r t h}{(1-p q h)^{2}}
$$

Finally, we must make grow simultaneously and identically all the lines of the newly created column, to obtain any plateau. Let us notice that there are $n+1$ different ways to add $n$ cells to make a line from a cell. It means that, for the last plateau, we substitute $p$ by $p^{n+1}, q$ by $q^{n+1}$ and $r$ by $r^{n+1}$, for all $n \geqslant 0$. Then, the generating function of the second case is:

$$
\sum_{n \geqslant 0}(n+1) H(t, 1,1, p, 1) \frac{p^{n+1} q^{n+1} r^{n+1} t h}{\left(1-p^{n+1} q^{n+1} h\right)^{2}} .
$$

- The previous growth creates polycubes that are not parallelograms, in particular when the last plateau has a too low bottom (decomposition 4 in Figure 8).

To begin, let us consider a parallelogram polycube. We stick at the back a column of height equal to the height of the last plateau, what means we add a new plateau. This is translated by substituting $h$ by $h p q$. Next, we add a unique cell under this column. This is translated by $H(t, h p q, 1, p, 1) p q r t h$. Then, we make the column grow upward and downward, what leads, as previously, to a factor $\frac{1}{(1-p q h)^{2}}$.

Finally, the simultaneous and identical growth of all the lines of the created column is generated by the substitution, for the last plateau, of $p$ by $p^{n+1}, q$ by $q^{n+1}$ and $r$ of $r^{n+1}$, for all $n \geqslant 0$. This case is enumerated by:

$$
\sum_{n \geqslant 0}(n+1) H\left(t, h p^{n+1} q^{n+1}, 1, p, 1\right) \frac{p^{n+1} q^{n+1} r^{n+1} t h}{\left(1-p^{n+1} q^{n+1} h\right)^{2}} .
$$

- We also have to remove polycubes such that last plateau has a too much forward front face (decomposition 5 in Figure 8).

We can notice that, compared wtih the case of upward growth, we simply have to invert the role of $r$ and $h$, what allows us to conclude that this case is enumerated by:

$$
\sum_{n \geqslant 0}(n+1) H\left(t, 1, r p^{n+1} q^{n+1}, p, 1\right) \frac{p^{n+1} q^{n+1} r t h^{n+1}}{\left(1-r p^{n+1} q^{n+1}\right)^{2}} .
$$

- Computing the above expression, we have removed twice the polycubes such that their last plateau has a bottom too low and a front face too much forward (decomposition 6 in Figure 8). So, we have to add their generating function.

One more time, let us consider a parallelogram polycube, enumerated by $H(t, h, r, p, q)$. First, we duplicate the last plateau, what is expressed by $H(t, h p q, r p q, p, 1) t$. Second, we add a line and a column to this plateau, what is enumerated by $H(t,(h+$ 1) $p q,(r+1) p q, p, 1) p q r t h$. Then, we make grow upward and backward the plateau, which leads, for the last plateau, to substitute $p$ by $p^{n+1}, q$ by $q^{n+1}$ and $r$ by $r^{n+1}$, for all $n \geqslant 0$ : 


$$
\sum_{n \geqslant 0}(n+1) H\left(t,\left(h^{n+1}+1\right) p^{n+1} q^{n+1},(r+1) p q, p, 1\right) p^{n+1} q^{n+1} r t h^{n+1} .
$$

Thus, the upward and backward growth allows us to find the following generating function:

$$
\begin{aligned}
\sum_{m \geqslant 0} \sum_{n \geqslant 0} & (m+1)(n+1) t r^{m+1} h^{n+1}(p q)^{n+m+1} \\
& \times H\left(t,\left(h^{n+1}+1\right) p^{n+1} q^{n+1},\left(r^{m+1}+1\right) p^{m+1} q^{m+1}, p, 1\right) .
\end{aligned}
$$

Proposition 8. The generating function of the parallelogram polycubes with respect to volume, width, area of the rightmost face, heigth of the last plateau and depth satisfies:

$$
\begin{aligned}
H(t, h, r, p, q) & =P l(t, h, r, p, q) \\
& +\sum_{n \geqslant 0}(n+1) H(t, 1,1, p, 1) \frac{p^{n+1} q^{n+1} r^{n+1} t h}{\left(1-p^{n+1} q^{n+1} h\right)^{2}} \\
& -\sum_{n \geqslant 0}(n+1) H\left(t, h p^{n+1} q^{n+1}, 1, p, 1\right) \frac{p^{n+1} q^{n+1} r^{n+1} t h}{\left(1-p^{n+1} q^{n+1} h\right)^{2}} \\
& -\sum_{n \geqslant 0}(n+1) H\left(t, 1, r p^{n+1} q^{n+1}, p, 1\right) \frac{p^{n+1} q^{n+1} r t h^{n+1}}{\left(1-r p^{n+1} q^{n+1}\right)^{2}} \\
& +\sum_{m \geqslant 0} \sum_{n \geqslant 0}(m+1)(n+1) t r^{m+1} h^{n+1}(p q)^{n+m+1} \\
& \times H\left(t,\left(h^{n+1}+1\right) p^{n+1} q^{n+1},\left(r^{m+1}+1\right) p^{m+1} q^{m+1}, p, 1\right)
\end{aligned}
$$

Unfortunately, we did not succeed in solving the previous functional equation. As far as we know, there is, at the moment, no method allowing us to find an expression of $H(t, p)$. However, it has been possible to extend some properties of $P(t, p$,$) to H(t, p)$. Their characterization is summed up in the following proposition:

\section{Proposition 9.}

1. $h_{n, n}=1$;

2. $h_{1, n}=\tau(n)$;

3. $h_{n-1, n}=2(n-1)$;

4. $h_{n-2, n}=2 n^{2}-6 n+2$;

4. $h_{n-3, n}=\frac{4}{3} n^{3}-8 n^{2}+\frac{23}{3} n+13$.

The proof is similar as the one of Proposition 5. Since the values of $h_{n-i, n}$ depend on the values of $\tau(i)$ it is not possible to find a general formula. However, with a similar proof as for Proposition 6 it is possible to find the asymptotic value of the polynomial:

Proposition 10. $h_{n-i, n}$ is a polynomial in $\mathbb{Q}[n]$ whose highest-degree-term is equal to $\frac{2^{i}}{i !} n^{i}$ 


\section{$5 \quad$ Asymptotic $d$-dimensional results}

We first generalize some parameters and definitions from 3-dimensional polycubes to $d$-dimensional ones, with $d \geqslant 4$. Then we give some asymptotic results concerning three new families of $d$-dimensional polycubes. Let us remark that these results extend the ones established in [10] in the case of specific $d$-dimensional polycubes.

\subsection{The extension to $d$-dimensional polycubes}

Let us now consider $\mathbb{N}^{d}$, with $d \geqslant 3$. An elementary cell is a unitary $d$-cube. A $d$-polycube is then a $d$-face connected finite set of elementary cells, defined up to a translation. The volume of a $d$-polycube is the number of its elementary cells.

Let $\left(0, \overrightarrow{i_{1}}, \ldots, \overrightarrow{i_{d}}\right)$ be an orthonormal coordinate system. The width (resp. height) of a $d$-polycube is the difference between its greatest index and its smallest index according to $\overrightarrow{i_{1}}$ (resp. $\overrightarrow{i_{2}}$ ). A $d$-polycube is said to be vertically-convex if its intersection with any hyperplane $\left(\overrightarrow{i_{2}}, \ldots, \overrightarrow{i_{d}}\right)$ is a vertically-convex $(d-1)$-polycube. It is said horizontallyconvex if its intersection with any hyperplane $\left(\overrightarrow{i_{1}}, \overrightarrow{i_{3}}, \overrightarrow{i_{4}}, \ldots, \overrightarrow{i_{d}}\right)$ is a horizontally-convex $(d-1)$-polycube. Other convexities can be defined, but they are not useful here.

The notion of step is extended as follows. A step in direction $j$ is a positive move of one unit along the axis $\overrightarrow{i_{j}}$. Then a directed $d$-polycube is such that each cell can be reached from a distinguished one, the root, by a path only made of steps in direction $j$, with $1 \leqslant j \leqslant d$.

A $d$-stratum of a $d$-polycube is a $d$-polycube of width 1 . We will say stratum instead of $d$-stratum if there is no ambiguity. For $d \geqslant 3$, the rightmost face of the $i$ th stratum is the $d$-face of this stratum on which is glued the $(i+1)^{t h}$ stratum and its surface is the number of its cells. The $(i+1)^{t h}$ stratum is called the rightmost stratum of the $i^{\text {th }}$ one.

A $s$-d-polycube is a $d$-polycube that can be split into strata. A pseudo-directed $d$ polycube is a $s$ - $d$-polycube such that each of its strata has to be glued to the previous one using a distinguished cell that must coincide with any cell of the previous stratum. The distinguished cell of a stratum can be, for instance, the lowest cell of the first column. In fact, the distinguished cell plays the same role as the root in the case of directed polycubes. Even if the considered strata are not directed, they are glued according to a similar rule.

\section{$5.2 \quad$ Asymptotic results}

Let $d \geqslant 3$ and let $R^{d}(t, p)=\sum_{n, m \geqslant 0} R_{n, m}^{d} t^{n} p^{m}$ the generating function of directed s- $d$ polycubes with respect to width and volume.

Proposition 11. The expresssion $r_{n-i, n}^{d}$ is a polynomial in $\mathbb{Q}[n]$ whose highest-degreeterm is equal to $\frac{2^{i}(d-1)^{i}}{i !} n^{i}$.

Proof. The proof is essentially based on the fact that adding a cell to a stratum of a directed $s-d$-polycube is equivalent to inject a cell into a directed s- $(d-1)$-polycube. As 
a consequence, there is a unique possibility to add a cell to a $d$-stratum of volume 1 with respect to each of the $d-1$ directions. Then, the rightmost stratum initially glued to the modified stratum can be glued again in two different ways in order to satisfy the property of direction of directed s- $d$-polycubes. Note that the last stratum is not concerned by this construction since it has no rightmost stratum. So, there are $2(d-1)$ possible constructions for the $i$ selected strata, which leads to $(2(d-1))^{i}\left(\begin{array}{c}n-i-1 \\ i\end{array}\right)$ possibilities to realize this operation. If the last stratum belongs to the set of selected strata, there are only $2^{i} \times(d-1)^{i-1}\left(\begin{array}{c}n-i-2 \\ i\end{array}\right)$ possibilities. In all the other cases, there are $\left(\begin{array}{c}n-i-1 \\ i-j\end{array}\right)$ or $\left(\begin{array}{c}n-i-2 \\ i-j\end{array}\right)$ (and $j>1$ ) possibilities to choose strata, leading to a term of degree $i-j$. Since $j>1$, the highest-degree-term in $O\left(n^{i-j}\right)$ is unimportant with respect to $n^{i}$. Finally, the highest-degree-term of $r_{n-i, n}^{d}$ is equal to $\frac{2^{i}(d-1)^{i}}{i !} n^{i}$.

Let $U^{d}(t, p)=\sum_{n, m \geqslant 0} u_{n, m}^{d} t^{n} p^{m}$ the generating function of directed s-polycubes with respect to width and volume. With a similar reasoning, it is also possible to prove the following result:

Proposition 12. The expresssion $u_{n-i, n}^{d}$ is a polynomial in $\mathbb{Q}[n]$ whose highest-degreeterm is equal to $\frac{2^{i}(d-1)^{i}}{i !} n^{i}$.

Note that, in [10], the same asymptotic result appears for another class of polycubes, the rs-directed $d$-polycubes. Moreover, the Propositions 11 and 12 generalize known results in dimension $3[10]$.

Let us now adress the case of s- $d$-polycubes. Let $V^{d}(t, p)=\sum_{n, m \geqslant 0} v_{n, m}^{d} t^{n} p^{m}$ their generating function with respect to width and volume.

Proposition 13. The expresssion $v_{n-i, n}^{d}$ is a polynomial in $\mathbb{Q}[n]$ whose highest-degreeterm is of the form $\frac{\alpha 2^{i}(d-1)^{i}}{i !} n^{i}$ where $\alpha$ is a power of 2 such that $1 \leqslant \alpha \leqslant 2^{i}$.

Proof. The difference with proofs of Propositions 11 and 13 results from the gluing of modified strata. As the considered $d$-polycube is no more directed, there are not necessarily two different ways to glue again the rightmost stratum initially glued to the modified stratum. If the rightmost stratum contains only one cell, there are effectively two ways. But if it contains two cells, there are four ways of gluing, which leads to the proposition.

\section{Conclusion}

After introducing criteria to easily characterize classes of polycubes, we have defined a new directed family, the plateau polycubes, that we have enumerated with respect to volume and width. We also have exhibited a subclass: the parallelogram polycubes. Even though we did not find an expression of their generating function, it has been 
possible to extend to these polycubes the main properties found for the plateau polycubes. Moreover, this work has also allowed us to extend to any dimension two asymptotic results valid for some classes of 3-dimensional polycubes and to find another one concerning the enumeration of $d$-dimensional polycubes $(d \geqslant 3)$ that can be split into strata. We currently work on how to refine the study of parallelogram polycubes and, more precisely, we expect to find a general expression for their generating function. We also expect to find formulae for new families of $\mathrm{s}-d$-polycubes.

\section{References}

[1] M. Abramowitz, I. A. Stegun, eds., Handbook of Mathematical Functions, National Bureau of Standards Applied Math. Series 55 (1964), 840.

[2] G. Aleksandrowicz, G. Barequet, Counting $d$-dimensional polycubes and nonrectangular planar polyominoes, in Proc: 12th Ann. Int. Computing Combinatorics Conf., Taipei, Taiwan in: Lecture Notes in Computer Science, vol. 4112, Spinger-Verlag (2006), 418-427.

[3] E.A. Bender, D.E. Knuth, Enumeration of Plane Partitions, J. Combin. Theory Ser. A. 13 (1972), 40-54.

[4] Bressoud, D.M., Proofs and Confirmations: The Story of the Alternating Sign Matrix Conjecture, Spectrum Series, Cambridge University Press (1999).

[5] S.R. Broadbent, J.M. Hammersley, Percolation processes; I. Crystals and mazes, Proc. Camb. Philos. Soc. 53 (1957),629-641.

[6] M. Bousquet-Mélou, A method for the enumeration of various classes of columnconvex polygons, Discrete Mathematics, vol 154 (1996), 4-25.

[7] B. Blanpain, J.-M. Champarnaud, J.-Ph. Dubernard, Geometrical Languages, LATA 2007, Report 35/07, GRLMC Universitat Rovira I Virgili 52007, 127-138.

[8] J.-M. Champarnaud, J.-Ph. Dubernard, H. Jeanne, An efficient algorithm to test whether a binary and prolongeable language is geometrical, Int. J. Found. Comput. Sci.Vol. 20(4) (2009), 763-774.

[9] J.-M. Champarnaud, J.-Ph. Dubernard, H. Jeanne, Enumeration of specific classes of polycubes, Lattice Path Combinatorics and Applications, Siena, Italy (2010), 79-87.

[10] J.-M. Champarnaud, J.-Ph. Dubernard, H. Jeanne, A generic method for the enumeration of various classes of directed polycubes, Discrete Mathematics \& Theoretical Computer Science 15:1 (2013), 183-200.

[11] H. Cohn, M. Larsen, J. Propp, The Shape of a Typical Boxed Plane Partition, New York J. Math. 4 (1998), 137-166.

[12] J.-Ph. Dubernard, Énumérations, Automates et Langages Géométriques, Mémoire d'habilitation à diriger des recherches, Université de Rouen, 25 Novembre 2011.

[13] S. Feretic, A q-enumeration of convex polyominoes by festoon approach, Theoret. Comput. Sci., 319(1-3) (2004), 333-356. 
[14] H. Jeanne, Langages géométriques et polycubes, $\mathrm{PhD}$ thesis, Université de Rouen (2010).

[15] G. Largeteau, Quantification du taux d'invalidité d'applications temps-réel à contraintes strictes, PhD Thesis, Université de Poitiers, 2004.

[16] G. Largeteau, D. Geniet, Quantification du taux d'invalidité d'applications tempsréel à contraintes strictes, Tech. et Sci. Inf. 27(5) (2008), 589-625

[17] W.F. Lunnon, Counting polyominoes, in: A.O.L. Atkin B.J. Birch (Eds), Computers in Number Theory, Academic Press, London (1971), 347-372.

[18] W.F. Lunnon, Symmetry of cubical and general polyominoes, in: R.C. Read (Eds), Graph Theory and Computing, Academic Press, London (1972), 101-109

[19] Stanley, R.P., Enumerative combinatorics. 2, chapter 7, Cambridge Studies in Advanced Math, Wadsworth \& Brooks/Cole Advanced Books \& Software (2001).

[20] The On-Line Encyclopedia of Integer Sequences, http://oeis.org/, sequence A000005. 